\title{
Integrated Disease Management against Wilt Disease of Pigeonpea Caused by Fusarium oxysporum f. sp. udum
}

\author{
P.H. Ghante*, A.P. Suryawanshi, K.M. Kanase, S.D. Somwanshi and D.S. Thaware \\ Department of Plant Pathology, College of Agriculture, Parbhani, Vasantrao Naik \\ Marathwada Krishi Vidyapeeth, Parbhani 431402 (M.S), India \\ *Corresponding author
}

\section{A B S T R A C T}

\begin{tabular}{|l|}
\hline Ke y w o r d s \\
$\begin{array}{l}\text { Pigeonpea wilt, Fusarium } \\
\text { oxysporum f. sp. } \text { udum, in } \\
\text { vivo, Integrated disease } \\
\text { management, } \\
\text { Azoxystrobin and soil } \\
\text { drenching }\end{array}$ \\
\hline Article Info \\
\hline $\begin{array}{l}\text { Accepted: } \\
\text { 15 September } 2018 \\
\text { Available Online: } \\
\text { 10 October } 2018\end{array}$ \\
\hline
\end{tabular}

\section{Introduction}

Pigeonpea [Cajanus cajan (L.) Millspaugh] is one of the most important pulse crops in the semi-arid tropics. It used as food as well as vegetable protein source and of fodder.

Endowed with excellent food and fodder qualities, these crops also restore soil fertility by scavenging atmospheric nitrogen, adding organic matter, enhancing phosphorus availability as well as improving physical, chemical and biological properties of the soil.The largest producer of pigeonpea in the

\begin{abstract}
Pigeonpea wilt caused by Fusarium oxysporum f. sp. udum is one of the most devastating soil-borne diseases of Pigeonpea. Concerned study conducted during Kharif 2016 and Kharif 2017 in Agricultural Research Station, Badnapur (M.S) aimed to find integrated disease management strategies to control Fusarium oxysporum f. sp. udum causing pigeonpea wilt disease. Result indicated that all 16 treatments during Kharif 2016 under normal soil and sick soil condition, soil application of $(T$. viride + neem seed cake $)+$ seed treatment of (carbendazim $25 \% \mathrm{WP}+$ mancozeb $50 \% \mathrm{WP}+T$. viride $)+$ soil drenching of azoxystrobin $23 \mathrm{EC}$ showed minimum wilt incidence i.e. $3.33 \%$ and $40.83 \%$ with maximum yield compared to other treatments i.e. $1424.28 \mathrm{~kg} / \mathrm{ha}$ and $513.05 \mathrm{~kg} / \mathrm{ha}$, respectively. During Kharif 2017 under normal soil and sick soil conditions, soil application of $(T$. viride + neem seed cake) + seed treatment of (carbendazim $25 \% \mathrm{WP}+$ mancozeb $50 \% \mathrm{WP}+T$. viride $)+$ soil drenching of azoxystrobin $23 \mathrm{EC}$ showed minimum wilt incidence i.e. $7.12 \%$ and $45.42 \%$ with maximum yield i.e. $1368.73 \mathrm{~kg} / \mathrm{ha}$ and 458.38 $\mathrm{kg} / \mathrm{ha}$, respectively compared to other treatments.
\end{abstract}

world is India, where it is widely cultivated with minimal input of nutrients and pest management measures. The main constraints in boosting the yield of the crop are susceptibility to diseases and insects.

The crop is attacked by more than 100 pathogens (Nene et al., 1996) including fungi, bacteria, viruses, phytoplasma like organisms and nematodes. However, only a few of them cause economic losses (Kannaiyan et al., 1984). The diseases of considerable economic importance at present are sterility mosaic, Fusarium wilt, Phytophthora blight, 
Macrophomina root rot, stem canker and Alternaria blight. Fusarium wilt is the most important disease of pigeonpea in India resulting in yield losses up to 67 per cent at maturity and 100 per cent in case of infection at pre-pod stage (Kannaiyan and Nene, 1981). The Fusarium wilt in pigeonpea was first reported from Bihar by Butler (1910).

The pathogen is primarily a soil inhabitant; hence controlling the disease is very difficult. Application of carbendazim has been successful in controlling the disease, but to a limited extent and also it is not economical. Bio-control approaches have been initiated by using antagonistic microorganisms to combat the wilt disease in pigeonpea. Many control measures have been suggested but, costeffective options for the management of this disease have not been developed. Keeping this in view, recent investigations were envisaged with the development of integrated management approaches for pigeonpea wilt disease.

\section{Materials and Methods}

The experiment was conducted at Agricultural Research Station, Badnapur VNMKV, Parbhani (M.S.) during Kharif 2016 and Kharif 2017. Based on in vitro (field) studies, the most effective five fungicides viz., carbendazim, carbendazim + mancozeb (for seed treatment), propiconazole, thiophanate methyl and azoxystrobin (for soil drenching) were selected for concerned study.

One bio-agent $T$. viride (for both seed treatment and soil application) and two organic amendments viz., neem seed cake and castor seed cake for (soil application) were selected and integrated alone as well as in combination to manage pigeonpea wilt ( $F$. $u d u m)$. The experiment was conducted for two consecutive years during Kharif 2015-16 and Kharif 2016-17.
The seed of susceptible pigeonpea cv. ICP 2376 were treated before sowing with the seed dressing fungicides and the bio-agent viz., $T$. viride. The soil application of test organic amendments and the test fungicides was done at 30 DAS.

Fungicide and bio-agent treated seeds of pigeonpea $\mathrm{cv}$. ICP-2376 were sown $(90 \mathrm{~cm} \mathrm{x}$ $20 \mathrm{~cm}$ ) in randomized plots (Gross plot Size: $13 \mathrm{~m}$ x $85 \mathrm{~m}$, Net plot Size: $12.8 \mathrm{~m}$ x $84 \mathrm{~m}$, Block size per treatment: $3.6 \mathrm{~m} \mathrm{x} 4 \mathrm{~m}$ with 4 rows and 20 plants / row on dated 12 June 2015 and 15 June 2016 for two Kharif seasons, respectively. The crop was grown by applying all recommended package of practices and irrigated as and when required.

\section{Results and Discussion}

\section{Efficacy of various treatments integration against wilt ( $F$. udum) incidence and seed yield during Kharif 2015-16}

Under normal soil and sick soil conditions, $\mathrm{T}_{16}$ [soil application of $(T$. viride + neem seed cake) + seed treatment of (carbendazim $25 \%$ $\mathrm{WP}+$ mancozeb $50 \% \mathrm{WP}+T$. viride $)+$ soil drenching of azoxystrobin 23 EC ] showed minimum wilt incidence i.e. 3.33 and $40.83 \%$ with maximum yield compared to other treatments i.e. 1424.28 and $513.05 \mathrm{~kg} / \mathrm{ha}$, respectively.

Second best treatment was $\mathrm{T}_{15}$ [soil application of $(T$. viride + neem seed cake $)+$ seed treatment of (carbendazim $25 \% \mathrm{WP}+$ mancozeb $50 \% \mathrm{WP}+T . v$.) + soil drenching of thiophanate methyl] showed $5 \%$ wilt incidence with $1347.48 \mathrm{~kg} / \mathrm{ha}$ in normal soil and under sick soil $43.93 \%$ wilt incidence and $491.58 \mathrm{~kg} / \mathrm{ha}$ yield were recorded. Maximum wilt incidences (43.92 and $100 \%$ ) were recorded in untreated control $\mathrm{T}_{17}$ with 582.33 $\mathrm{kg} / \mathrm{ha}$ yield and without any yield in normal and sick soil (Table 1), respectively. 
Table.1 Efficacy of various treatments integration against wilt (F. udum) incidence and seed yield in pigeonpea cv. ICP 2376 during Kharif 2015-16

\begin{tabular}{|c|c|c|c|c|c|c|c|c|}
\hline \multirow[t]{2}{*}{$\begin{array}{l}\text { Tr. } \\
\text { No. }\end{array}$} & \multirow[t]{2}{*}{ Treatments } & \multirow[t]{2}{*}{ Rate of application } & \multicolumn{2}{|c|}{ Wilt incidence (\%) } & \multicolumn{2}{|c|}{$\begin{array}{l}\text { Reduction over } \\
\text { control }(\%)\end{array}$} & \multicolumn{2}{|c|}{$\begin{array}{l}\text { Yield } \\
\text { (Kg/ha) }\end{array}$} \\
\hline & & & Normal soil & Sick soil & $\begin{array}{l}\text { Norm } \\
\text { al soil }\end{array}$ & $\begin{array}{l}\text { Sick } \\
\text { soil }\end{array}$ & $\begin{array}{l}\text { Norma } \\
\text { I soil }\end{array}$ & $\begin{array}{l}\text { Sick } \\
\text { soil }\end{array}$ \\
\hline $\mathbf{T}_{1}$ & Carbendazim $50 \%$ WP (ST) & $1 \mathrm{~g} / \mathrm{kg}$ seed & $25.83(30.48)$ & $85.37(67.64)$ & 41.19 & 14.63 & 735.93 & 120.18 \\
\hline $\mathbf{T}_{2}$ & Carbendazim $25 \% \mathrm{WP}+$ mancozeb50 \% WP (ST) & $3 \mathrm{~g} / \mathrm{kg}$ seed & $23.43(28.89)$ & $82.50(65.30)$ & 46.65 & 17.50 & 757.18 & 143.42 \\
\hline $\mathbf{T}_{3}$ & Trichoderma viride (ST) & $12 \mathrm{~g} / \mathrm{kg}$ seed & $27.50(31.56)$ & $87.08(69.09)$ & 37.39 & 12.92 & 710.04 & 100.04 \\
\hline $\mathbf{T}_{4}$ & Carbendazim $50 \%$ WP $(\mathrm{ST})+$ Trichoderma viride $(\mathrm{ST})$ & $1 \mathrm{~g} / \mathrm{kg}$ seed $+12 \mathrm{~g} / \mathrm{kg}$ seed & $22.92(28.57)$ & $80.21(63.71)$ & 47.81 & 19.79 & 766.48 & 151.17 \\
\hline $\mathbf{T}_{5}$ & $($ Carbendazim $25 \% \mathrm{WP}+$ mancozeb $50 \% \mathrm{WP})+T$. viride $(\mathrm{ST})$ & $3 \mathrm{~g} / \mathrm{kg}$ seed $+12 \mathrm{~g} / \mathrm{kg}$ seed & $21.25(27.39)$ & $77.08(61.38)$ & 51.62 & 22.92 & 780.20 & 160.91 \\
\hline $\mathbf{T}_{6}$ & Neem seed cake (SA) & $5 \mathrm{q} / \mathrm{ha}$ & $23.75(29.13)$ & $80.00(63.68)$ & 45.92 & 20.00 & 761.39 & 154.49 \\
\hline $\mathbf{T}_{7}$ & Castor seed cake (SA) & $5 \mathrm{q} / \mathrm{ha}$ & $24.17(29.40)$ & $83.28(65.95)$ & 44.97 & 16.72 & 740.36 & 140.55 \\
\hline $\mathbf{T}_{8}$ & Trichoderma viride (SA) & $20 \mathrm{~kg} / \mathrm{ha}$ & $21.25(27.39)$ & $78.33(62.38)$ & 51.62 & 21.67 & 793.04 & 171.09 \\
\hline $\mathbf{T}_{9}$ & Trichoderma viride $(\mathrm{SA})+$ neem cake $(\mathrm{SA})$ & $20 \mathrm{~kg} / \mathrm{ha}+5 \mathrm{q} / \mathrm{ha}$ & $17.50(24.66)$ & $71.09(57.60)$ & 60.15 & 28.91 & 912.11 & 223.10 \\
\hline $\mathbf{T}_{10}$ & Trichoderma viride (SA) + castor seed cake (SA) & $20 \mathrm{~kg} / \mathrm{ha}+5 \mathrm{q} / \mathrm{ha}$ & $19.25(25.98)$ & $76.25(60.92)$ & 56.17 & 23.75 & 832.21 & 187.69 \\
\hline $\mathbf{T}_{11}$ & $\begin{array}{l}\text { Trichoderma viride (SA) + [carbendazim } 25 \% \mathrm{WP}+\text { mancozeb } 50 \\
\% \mathrm{WP} \text { ] (ST) }\end{array}$ & $20 \mathrm{~kg} / \mathrm{ha}+3 \mathrm{~g} / \mathrm{kg}$ seed & $15.42(23.00)$ & $69.43(56.79)$ & 64.89 & 30.57 & 958.15 & 253.43 \\
\hline $\mathbf{T}_{12}$ & $\begin{array}{l}\text { T.v. }(\mathrm{SA})+\text { neem seed cake }(\mathrm{SA})+[\text { carbendazim } 25 \% \mathrm{WP}+ \\
\text { mancozeb } 50 \% \mathrm{WP}](\mathrm{ST})+\text { T.v. }(\mathrm{ST})\end{array}$ & $\begin{array}{l}20 \mathrm{~kg} / \mathrm{ha}+5 \mathrm{q} / \mathrm{ha}+3 \mathrm{~g} / \mathrm{kg} \text { seed } \\
+12 \mathrm{~g} / \mathrm{kg} \text { seed }\end{array}$ & $12.50(20.63)$ & $62.50(52.39)$ & 71.54 & 37.50 & $\begin{array}{l}1000.6 \\
5\end{array}$ & 305.00 \\
\hline $\mathbf{T}_{13}$ & $\begin{array}{l}\text { T.v. }(\mathrm{SA})+\text { neem seed cake }(\mathrm{SA})+[\text { carbendazim + mancozeb] }(\mathrm{ST}) \\
+ \text { T.v. }(\mathrm{ST})+[\text { carbendazim } 25 \% \mathrm{WP}+\text { mancozeb } 50 \% \mathrm{WP}](\mathrm{SD})\end{array}$ & $\begin{array}{l}20 \mathrm{~kg} / \mathrm{ha}+5 \mathrm{q} / \mathrm{ha}+3 \mathrm{~g} / \mathrm{kg} \text { seed }+ \\
12 \mathrm{~g} / \mathrm{kg} \text { seed }+@ 0.25 \%\end{array}$ & $9.58(17.88)$ & $49.08(44.46)$ & 78.19 & 50.92 & $\begin{array}{l}1109.1 \\
0\end{array}$ & 426.29 \\
\hline $\mathbf{T}_{14}$ & $\begin{array}{l}\text { T.v. }(\mathrm{SA})+\text { neem seed cake }(\mathrm{SA})+[\text { carbendazim } 25 \% \mathrm{WP}+ \\
\text { mancozeb } 50 \% \mathrm{WP}](\mathrm{ST})+\text { T.v. }(\mathrm{ST})+\text { propiconazole }(\mathrm{SD})\end{array}$ & $\begin{array}{l}20 \mathrm{~kg} / \mathrm{ha}+5 \mathrm{q} / \mathrm{ha}+3 \mathrm{~g} / \mathrm{kg} \text { seed }+ \\
12 \mathrm{~g} / \mathrm{kg} \text { seed }+@ 0.1 \%\end{array}$ & $7.13(15.18)$ & $46.25(42.82)$ & 83.77 & 53.75 & $\begin{array}{l}1175.9 \\
4\end{array}$ & 457.27 \\
\hline $\mathbf{T}_{15}$ & $\begin{array}{l}\text { T.v. }(\mathrm{SA})+\text { neem seed cake }(\mathrm{SA})+[\text { carbendazim } 25 \% \mathrm{WP}+ \\
\text { mancozeb } 50 \% \mathrm{WP}](\mathrm{ST})+\text { T.v. }(\mathrm{ST})+\text { thiophanate methyl }(\mathrm{SD})\end{array}$ & $\begin{array}{l}20 \mathrm{~kg} / \mathrm{ha}+5 \mathrm{q} / \mathrm{ha}+3 \mathrm{~g} / \mathrm{kg} \text { seed }+ \\
12 \mathrm{~g} / \mathrm{kg} \text { seed }+0.1 \%\end{array}$ & $5.00(12.00)$ & $43.93(41.46)$ & 88.62 & 56.07 & $\begin{array}{l}1347.4 \\
8\end{array}$ & 491.58 \\
\hline$T_{16}$ & $\begin{array}{l}\text { T.v. }(\mathrm{SA})+\text { neem seed cake }(\mathrm{SA})+[\text { carbendazim } 25 \% \mathrm{WP}+ \\
\text { mancozeb } 50 \% \mathrm{WP}](\mathrm{ST})+\text { T.v. }(\mathrm{ST})+\text { azoxystrobin } 23 \mathrm{EC}(\mathrm{SD})\end{array}$ & $\begin{array}{l}20 \mathrm{~kg} / \mathrm{ha}+5 \mathrm{q} / \mathrm{ha}+3 \mathrm{~g} / \mathrm{kg} \text { seed }+ \\
12 \mathrm{~g} / \mathrm{kg} \text { seed }+0.1 \%\end{array}$ & $\begin{array}{l}3.33 \\
(8.33)\end{array}$ & $40.83(39.67)$ & 92.42 & 59.17 & $\begin{array}{l}1424.2 \\
8\end{array}$ & 513.05 \\
\hline $\mathbf{T}_{17}$ & Control & Untreated & $43.92(41.47)$ & $100(90.00)$ & 00.00 & 00.00 & 582.33 & 0.00 \\
\hline \multicolumn{3}{|c|}{ S.E. \pm} & 2.00 & 2.89 & -- & -- & 66.12 & 36.25 \\
\hline \multicolumn{3}{|c|}{ C.D. $(P=0.05)$} & 5.81 & 8.38 & -- & -- & 191.35 & 104.91 \\
\hline \multicolumn{3}{|c|}{ C.V. } & 14.00 & 8.48 & -- & -- & 12.65 & 16.69 \\
\hline
\end{tabular}

T. viride soil application: $20 \mathrm{~kg}$ talc carrier based T. viride mixed with $500 \mathrm{~kg}$ well decomposed FYM / ha.

ST: Seed Treatment; SA: Soil Application; SD: Soil Drenching.

Figures in parentheses are angular transformed values. 
Table.2 Efficacy of various treatments integration against wilt $(F . u d u m)$ incidence and seed yield in pigeonpea cv. ICP 2376 during Kharif 2016-17

\begin{tabular}{|c|c|c|c|c|c|c|c|c|}
\hline \multirow[t]{2}{*}{$\begin{array}{l}\text { Tr. } \\
\text { No. }\end{array}$} & \multirow[t]{2}{*}{ Treatments } & \multirow[t]{2}{*}{ Rate of application } & \multicolumn{2}{|c|}{ Wilt Incidence (\%) } & \multicolumn{2}{|c|}{$\begin{array}{l}\text { Reduction over } \\
\text { control }(\%)\end{array}$} & \multicolumn{2}{|l|}{$\begin{array}{l}\text { Yield } \\
\text { (kg/ha) }\end{array}$} \\
\hline & & & Normal soil & Sick soil & $\begin{array}{l}\text { Normal } \\
\text { soil }\end{array}$ & $\begin{array}{l}\text { Sick } \\
\text { soil }\end{array}$ & $\begin{array}{l}\text { Normal } \\
\text { soil }\end{array}$ & $\begin{array}{l}\text { Sick } \\
\text { soil }\end{array}$ \\
\hline $\mathbf{T}_{1}$ & Carbendazim $50 \%$ WP (ST) & $1 \mathrm{~g} / \mathrm{kg}$ seed & $28.33(32.10)$ & $89.17(70.93)$ & 41.89 & 10.83 & 685.91 & 57.33 \\
\hline $\mathbf{T}_{2}$ & Carbendazim $25 \% \mathrm{WP}+$ mancozeb $50 \% \mathrm{WP}(\mathrm{ST})$ & $3 \mathrm{~g} / \mathrm{kg}$ seed & $27.62(31.66)$ & $87.39(70.29)$ & 43.34 & 12.61 & 717.78 & 80.34 \\
\hline $\mathbf{T}_{3}$ & Trichoderma viride (ST) & $12 \mathrm{~g} / \mathrm{kg}$ seed & $29.17(32.60)$ & $91.25(73.87)$ & 40.16 & 08.75 & 662.23 & 44.93 \\
\hline $\mathbf{T}_{4}$ & Carbendazim $50 \%$ WP $(\mathrm{ST})+$ Trichoderma viride $(\mathrm{ST})$ & $1 \mathrm{~g} / \mathrm{kg}$ seed $+12 \mathrm{~g} / \mathrm{kg}$ seed & $26.25(30.79)$ & $84.17(66.66)$ & 46.15 & 15.83 & 734.83 & 102.48 \\
\hline $\mathbf{T}_{5}$ & $\begin{array}{l}\text { (Carbendazim } 25 \% \mathrm{WP}+\text { Mancozeb } 50 \% \mathrm{WP})+ \\
\text { Trichoderma viride }(\mathrm{ST})\end{array}$ & $\begin{array}{l}3 \mathrm{~g} / \mathrm{kg} \text { seed }+12 \mathrm{~g} / \mathrm{kg} \\
\text { seed }\end{array}$ & $25.00(29.93)$ & $82.03(65.02)$ & 48.72 & 17.97 & 742.13 & 119.52 \\
\hline $\mathbf{T}_{6}$ & Neem seed cake (SA) & $5 \mathrm{q} / \mathrm{ha}$ & $26.25(30.78)$ & $84.17(67.28)$ & 46.15 & 15.83 & 721.10 & 99.38 \\
\hline $\mathbf{T}_{7}$ & Castor seed cake (SA) & $5 \mathrm{q} / \mathrm{ha}$ & $27.92(31.87)$ & $86.60(69.85)$ & 42.73 & 13.40 & 692.77 & 83.44 \\
\hline $\mathbf{T}_{8}$ & Trichoderma viride (SA) & $20 \mathrm{~kg} / \mathrm{ha}$ & $24.26(29.45)$ & $83.75(66.33)$ & 50.24 & 16.25 & 756.96 & 121.95 \\
\hline $\mathbf{T}_{9}$ & Trichoderma viride $(\mathrm{SA})+$ neem seed cake $(\mathrm{SA})$ & $20 \mathrm{~kg} / \mathrm{ha}+5 \mathrm{q} / \mathrm{ha}$ & $21.67(27.72)$ & $75.25(60.31)$ & 55.55 & 24.75 & 853.68 & 168.66 \\
\hline $\mathbf{T}_{10}$ & Trichoderma viride $(\mathrm{SA})+$ castor seed cake (SA) & $20 \mathrm{~kg} / \mathrm{ha}+5 \mathrm{q} / \mathrm{ha}$ & $22.31(28.11)$ & $80.00(63.47)$ & 54.24 & 20.00 & 710.04 & 146.97 \\
\hline$T_{11}$ & $\begin{array}{l}\text { T. viride (SA) + [carbendazim } 25 \% \mathrm{WP}+\text { mancozeb } 50 \% \\
\text { WP] (ST) }\end{array}$ & $20 \mathrm{~kg} / \mathrm{ha}+3 \mathrm{~g} / \mathrm{kg}$ seed & $19.25(25.96)$ & $73.33(59.35)$ & 60.51 & 26.67 & 920.75 & 206.50 \\
\hline $\mathbf{T}_{12}$ & $\begin{array}{l}\text { T.v. }(\mathrm{SA})+\text { neem seed cake }(\mathrm{SA})+[\text { carbendazim } 25 \% \\
\mathrm{WP}+\text { mancozeb } 50 \% \mathrm{WP}](\mathrm{ST})+\text { T.v. }(\mathrm{ST})\end{array}$ & $\begin{array}{l}20 \mathrm{~kg} / \mathrm{ha}+5 \mathrm{q} / \mathrm{ha}+3 \mathrm{~g} / \\
\mathrm{kg} \text { seed }+12 \mathrm{~g} / \mathrm{kg} \text { seed }\end{array}$ & $16.31(23.75)$ & $66.55(54.84)$ & 66.54 & 33.45 & 969.22 & 258.30 \\
\hline $\mathbf{T}_{13}$ & $\begin{array}{l}\text { T.v. }(\mathrm{SA})+\text { neem seed cake }(\mathrm{SA})+[\text { carbendazim }+ \\
\text { mancozeb] }(\mathrm{ST})+\text { T.v. }(\mathrm{ST})+[\text { carbendazim } 25 \% \mathrm{WP}+ \\
\text { mancozeb } 50 \% \mathrm{WP}](\mathrm{SD})\end{array}$ & $\begin{array}{l}20 \mathrm{~kg} / \mathrm{ha}+5 \mathrm{q} / \mathrm{ha}+3 \mathrm{~g} / \\
\mathrm{kg} \mathrm{seed}+12 \mathrm{~g} / \mathrm{kg} \text { seed }+ \\
\text { @ } 0.25 \%\end{array}$ & $12.92(20.99)$ & $53.33(46.99)$ & 73.50 & 46.67 & 1077.45 & 387.78 \\
\hline$T_{14}$ & $\begin{array}{l}\text { T.v. }(\mathrm{SA})+\text { neem seed cake }(\mathrm{SA})+[\text { carbendazim } 25 \% \mathrm{WP} \\
+ \text { mancozeb } 50 \% \mathrm{WP}](\mathrm{ST})+\text { T.v. }(\mathrm{ST})+\text { propiconazole } \\
(\mathrm{SD})\end{array}$ & $\begin{array}{l}20 \mathrm{~kg} / \mathrm{ha}+5 \mathrm{q} / \mathrm{ha}+3 \mathrm{~g} / \\
\mathrm{kg} \text { seed }+12 \mathrm{~g} / \mathrm{kg} \text { seed }+ \\
@ 0.1 \%\end{array}$ & $10.47(18.77)$ & $51.20(45.67)$ & 78.52 & 48.80 & 1152.93 & 416.33 \\
\hline $\mathbf{T}_{15}$ & $\begin{array}{l}\text { T.v. }(\mathrm{SA})+\text { Neem seed cake (SA)+ [carbendazim } 25 \% \\
\text { WP + mancozeb } 50 \% \text { WP] }(\mathrm{ST})+\text { T.v. }(\mathrm{ST})+\text { thiophanate } \\
\text { methyl (SD) }\end{array}$ & $\begin{array}{l}20 \mathrm{~kg} / \mathrm{ha}+5 \mathrm{q} / \mathrm{ha}+3 \mathrm{~g} / \\
\mathrm{kg} \text { seed }+12 \mathrm{~g} / \mathrm{kg} \text { seed }+ \\
@ 0.1 \%\end{array}$ & $9.17(17.56)$ & $47.08(43.30)$ & 81.19 & 52.92 & 1336.85 & 443.77 \\
\hline $\mathbf{T}_{16}$ & $\begin{array}{l}\text { T.v. (SA) + Neem seed cake (SA)+ [Carbendazim } 25 \% \\
\text { WP + Mancozeb } 50 \% \text { WP] (ST) + T.v. (ST) + } \\
\text { azoxystrobin } 23 \text { EC (SD) }\end{array}$ & $\begin{array}{l}20 \mathrm{~kg} / \mathrm{ha}+5 \mathrm{q} / \mathrm{ha}+3 \mathrm{~g} / \\
\mathrm{kg} \mathrm{seed}+12 \mathrm{~g} / \mathrm{kg} \text { seed }+ \\
@ 0.1 \%\end{array}$ & $7.12(15.37)$ & $45.42(42.30)$ & 85.39 & 54.58 & 1368.73 & 458.38 \\
\hline $\mathbf{T}_{17}$ & Control & Untreated & $48.75(44.26)$ & $100(90.00)$ & 00.00 & 00.00 & 541.82 & 0.00 \\
\hline \multicolumn{3}{|c|}{ S.E. \pm} & 1.47 & 3.63 & -- & -- & 50.61 & 44.07 \\
\hline \multicolumn{3}{|c|}{ C.D. $(P=0.05)$} & 4.26 & 10.49 & -- & - & 146.44 & 127.52 \\
\hline \multicolumn{3}{|c|}{ C.V. } & 09.19 & 10.11 & -- & -- & 10.17 & 20.46 \\
\hline
\end{tabular}

Figures in parentheses are angular transformed values

ST: Seed Treatment; SA: Soil Application; SD: Soil Drenching. 
Table.3 Effect of various treatments integration on pooled mean (2015-16 and 2016-17) wilt incidence and seed yield in pigeonpea cv. ICP 2376

\begin{tabular}{|c|c|c|c|c|c|c|c|}
\hline \multirow{2}{*}{$\begin{array}{l}\text { Tr. } \\
\text { No. }\end{array}$} & \multirow{2}{*}{\multicolumn{2}{|c|}{ Treatments }} & \multirow[t]{2}{*}{ Rate of application } & \multicolumn{2}{|c|}{ Normal Soil } & \multicolumn{2}{|c|}{ Sick soil } \\
\hline & & & & $\begin{array}{c}\text { Wilt } \\
\text { incidence } \\
*(\%)\end{array}$ & $\begin{array}{l}\text { Yield } \\
\mathrm{kg} / \mathrm{ha}\end{array}$ & $\begin{array}{l}\text { Wilt incidence } \\
*(\%)\end{array}$ & $\begin{array}{l}\text { Yield } \\
\text { kg/ha }\end{array}$ \\
\hline $\mathbf{T}_{1}$ & \multicolumn{2}{|c|}{ Carbendazim $50 \%$ WP (ST) } & $1 \mathrm{~g} / \mathrm{kg}$ seed & $27.08(31.32)$ & 710.92 & $87.27(69.29)$ & 88.75 \\
\hline $\mathbf{T}_{2}$ & \multicolumn{2}{|c|}{ Carbendazim $25 \% \mathrm{WP}+$ mancozeb $50 \% \mathrm{WP}(\mathrm{ST})$} & $3 \mathrm{~g} / \mathrm{kg}$ seed & $25.52(30.28)$ & 737.48 & $84.95(67.79)$ & 111.88 \\
\hline $\mathbf{T}_{3}$ & \multicolumn{2}{|c|}{ Trichoderma viride (ST) } & $12 \mathrm{~g} / \mathrm{kg}$ seed & $28.33(32.08)$ & 686.13 & $89.17(71.48)$ & 72.49 \\
\hline $\mathbf{T}_{4}$ & \multicolumn{2}{|c|}{ Carbendazim $50 \%$ WP (ST) + Trichoderma viride (ST) } & $1 \mathrm{~g} / \mathrm{kg}$ seed $+12 \mathrm{~g} / \mathrm{kg}$ seed & $24.58(29.71)$ & 750.65 & $82.19(65.19)$ & 126.82 \\
\hline $\mathbf{T}_{5}$ & \multicolumn{2}{|c|}{ (Carbendazim $25 \% \mathrm{WP}+$ mancozeb $50 \% \mathrm{WP})+$ Trichoderma viride $(\mathrm{ST})$} & $3 \mathrm{~g} / \mathrm{kg}$ seed $+12 \mathrm{~g} / \mathrm{kg}$ seed & $23.13(28.66)$ & 761.17 & $79.56(63.20)$ & 140.21 \\
\hline $\mathbf{T}_{6}$ & \multicolumn{2}{|c|}{ Neem seed cake (SA) } & $5 \mathrm{q} / \mathrm{ha}$ & $25.00(29.96)$ & 741.25 & $82.08(65.48)$ & 126.93 \\
\hline $\mathbf{T}_{7}$ & \multicolumn{2}{|c|}{ Castor seed cake (SA) } & $5 \mathrm{q} / \mathrm{ha}$ & $26.04(30.64)$ & 716.57 & $84.94(67.90)$ & 111.99 \\
\hline $\mathbf{T}_{8}$ & \multicolumn{2}{|c|}{ Trichoderma viride (SA) } & $20 \mathrm{~kg} / \mathrm{ha}$ & $22.76(28.42)$ & 775.00 & $81.04(64.36)$ & 146.52 \\
\hline $\mathbf{T}_{9}$ & \multicolumn{2}{|c|}{ Trichoderma viride $(\mathrm{SA})+$ neem seed cake $(\mathrm{SA})$} & $20 \mathrm{~kg} / \mathrm{ha}+5 \mathrm{q} / \mathrm{ha}$ & $19.58(26.19)$ & 882.90 & $73.17(58.96)$ & 195.88 \\
\hline $\mathbf{T}_{10}$ & \multicolumn{2}{|c|}{ Trichoderma viride (SA) + castor seed cake (SA) } & $20 \mathrm{~kg} / \mathrm{ha}+5 \mathrm{q} / \mathrm{ha}$ & $20.78(27.04)$ & 771.13 & $78.13(62.20)$ & 167.33 \\
\hline $\mathbf{T}_{11}$ & \multicolumn{2}{|c|}{$\begin{array}{l}\text { Trichoderma viride }(\mathrm{SA})+\text { [carbendazim } 25 \% \mathrm{WP}+\text { mancozeb } 50 \% \mathrm{WP}] \\
\text { (ST) }\end{array}$} & $20 \mathrm{~kg} / \mathrm{ha}+3 \mathrm{~g} / \mathrm{kg}$ seed & $17.33(24.48)$ & 939.45 & $71.38(58.07)$ & 229.97 \\
\hline $\mathbf{T}_{12}$ & \multicolumn{2}{|c|}{$\begin{array}{l}\text { T.v. (SA) + neem seed cake (SA) + [carbendazim } 25 \% \mathrm{WP}+\text { mancozeb } 50 \% \\
\text { WP] (ST) + T.v. }(\mathrm{ST})\end{array}$} & $\begin{array}{l}20 \mathrm{~kg} / \mathrm{ha}+5 \mathrm{q} / \mathrm{ha}+3 \mathrm{~g} / \mathrm{kg} \\
\text { seed }+12 \mathrm{~g} / \mathrm{kg} \text { seed }\end{array}$ & $14.41(22.19)$ & 984.93 & $64.53(53.62)$ & 281.65 \\
\hline $\mathbf{T}_{13}$ & \multicolumn{2}{|c|}{$\begin{array}{l}\text { T.v. }(\mathrm{SA})+\text { Neem seed cake }(\mathrm{SA})+[\text { carbendazim + mancozeb }](\mathrm{ST})+T . v . \\
(\mathrm{ST})+[\text { carbendazim } 25 \% \mathrm{WP}+\text { mancozeb } 50 \% \mathrm{WP}](\mathrm{SD})\end{array}$} & $\begin{array}{l}20 \mathrm{~kg} / \mathrm{ha}+5 \mathrm{q} / \mathrm{ha}+3 \mathrm{~g} / \mathrm{kg} \text { seed } \\
+12 \mathrm{~g} / \mathrm{kg} \text { seed }+0.25 \%\end{array}$ & $11.25(19.44)$ & 1093.28 & $51.21(45.73)$ & 407.03 \\
\hline $\mathbf{T}_{14}$ & \multicolumn{2}{|c|}{$\begin{array}{l}\text { T.v. (SA) + neem seed cake (SA)+ [carbendazim } 25 \% \mathrm{WP}+\text { mancozeb } 50 \% \\
\text { WP] (ST) + T.v. }(\mathrm{ST})+\text { propiconazole (SD) }\end{array}$} & $\begin{array}{l}20 \mathrm{~kg} / \mathrm{ha}+5 \mathrm{q} / \mathrm{ha}+3 \mathrm{~g} / \mathrm{kg} \\
\text { seed }+12 \mathrm{~g} / \mathrm{kg} \text { seed }+@ 0.1 \%\end{array}$ & $8.80(16.98)$ & 1164.43 & $48.72(44.25)$ & 436.80 \\
\hline $\mathbf{T}_{15}$ & \multicolumn{2}{|c|}{$\begin{array}{l}\text { T.v. }(\mathrm{SA})+\text { Neem seed cake }(\mathrm{SA})+[\text { carbendazim } 25 \% \mathrm{WP}+\text { mancozeb } 50 \% \\
\text { WP] }(\mathrm{ST})+\text { T.v. }(\mathrm{ST})+\text { thiophanate methyl }(\mathrm{SD})\end{array}$} & $\begin{array}{l}20 \mathrm{~kg} / \mathrm{ha}+5 \mathrm{q} / \mathrm{ha}+3 \mathrm{~g} / \mathrm{kg} \\
\text { seed }+12 \mathrm{~g} / \mathrm{kg} \text { seed }+@ 0.1 \%\end{array}$ & $7.08(14.78)$ & 1342.17 & $45.51(42.38)$ & 467.68 \\
\hline $\mathbf{T}_{16}$ & \multicolumn{2}{|c|}{$\begin{array}{l}\text { T.v. (SA) + neem seed cake (SA)+ [carbendazim } 25 \% \mathrm{WP}+\text { mancozeb } 50 \% \\
\text { WP] (ST) + T.v. (ST) + azoxystrobin } 23 \mathrm{EC}(\mathrm{SD})\end{array}$} & $\begin{array}{l}20 \mathrm{~kg} / \mathrm{ha}+5 \mathrm{q} / \mathrm{ha}+3 \mathrm{~g} / \mathrm{kg} \text { seed } \\
+12 \mathrm{~g} / \mathrm{kg} \text { seed+ @ } 0.1 \%\end{array}$ & $5.23(11.85)$ & 1396.50 & $43.13(40.99)$ & 485.72 \\
\hline $\mathbf{T}_{17}$ & \multicolumn{2}{|l|}{ Control } & Untreated & $46.33(42.87)$ & 562.08 & $100.00(90.00)$ & 0.00 \\
\hline \multicolumn{4}{|c|}{ S.E. \pm} & 1.74 & 58.37 & 3.26 & 40.16 \\
\hline \multicolumn{4}{|c|}{ C.D. $(P=0.05)$} & 5.04 & 168.90 & 9.44 & 116.22 \\
\hline \multicolumn{4}{|c|}{ C.V. } & 11.59 & 11.41 & 9.29 & 18.58 \\
\hline \multirow{2}{*}{\multicolumn{2}{|c|}{ Factor (A=Year) }} & S.E. \pm & & 0.42 & 14.07 & 1.15 & 9.68 \\
\hline & & C.D. $(P=0.05)$ & & 1.19 & 39.82 & 3.24 & 27.39 \\
\hline \multirow{2}{*}{\multicolumn{2}{|c|}{ Factor $(B=$ Treatment) }} & S.E. \pm & & 1.24 & 41.02 & 3.34 & 28.22 \\
\hline & & C.D. $(P=0.05)$ & & 3.49 & 116.08 & 9.44 & 79.86 \\
\hline \multicolumn{4}{|c|}{ Factor (A X B) } & NS & NS & NS & NS \\
\hline
\end{tabular}

*Figures in parentheses are angular transformed values 
Table.4 Economics of integrated management practices imposed for Fusarium wilt disease of pigeonpea during Kharif 2015-16

\begin{tabular}{|c|c|c|c|c|c|c|c|c|c|c|c|}
\hline \multirow[t]{2}{*}{$\begin{array}{l}\text { Tr. } \\
\text { No. }\end{array}$} & \multirow[t]{2}{*}{ Treatments } & \multirow[t]{2}{*}{ Rate of application } & \multirow[t]{2}{*}{ PDI (\%) } & \multirow{2}{*}{$\begin{array}{l}\text { Seed } \\
\text { yield* }(\mathbf{k g} / \\
\text { ha) }\end{array}$} & \multirow{2}{*}{$\begin{array}{c}\text { Gross } \\
\text { returns^ } \\
(\text { Rs / ha) }\end{array}$} & \multirow{2}{*}{$\begin{array}{l}\text { Cost of } \\
\text { cultivation } \\
\text { (Rs/ha) }\end{array}$} & \multicolumn{2}{|c|}{$\begin{array}{l}\text { Cost plant protection (Rs } \\
\text { / ha) }\end{array}$} & \multirow[t]{2}{*}{$\begin{array}{l}\text { Total cost } \\
\text { (Rs / ha) }\end{array}$} & \multirow[t]{2}{*}{ Net profit } & \multirow[t]{2}{*}{ ICBR } \\
\hline & & & & & & & $\begin{array}{l}\text { Treat- } \\
\text { ments*** }\end{array}$ & $\begin{array}{l}\text { Labour } \\
\text { Charges }^{\wedge \wedge}\end{array}$ & & & \\
\hline 1 & 2 & 3 & 4 & 5 & 6 & 7 & 8 & 9 & 10 & 11 & 12 \\
\hline$T_{1}$ & Carbendazim $50 \%$ WP (ST) & $1 \mathrm{~g} / \mathrm{kg}$ seed & $25.83(30.48)$ & 735.93 & 34036.76 & 21697 & 18.40 & 180 & 21895.40 & 12141.36 & 1.55 \\
\hline $\mathrm{T}_{2}$ & $\begin{array}{l}\text { Carbendazim } 25 \% \mathrm{WP}+\text { mancozeb } 50 \% \mathrm{WP} \\
\text { (ST) }\end{array}$ & $3 \mathrm{~g} / \mathrm{kg}$ seed & $23.43(28.89)$ & 757.18 & 35019.58 & 21697 & 28.00 & 180 & 21905.00 & 13114.58 & 1.60 \\
\hline $\mathrm{T}_{3}$ & Trichoderma viride $(\mathrm{ST})$ & $12 \mathrm{~g} / \mathrm{kg}$ seed & $27.50(31.56)$ & 710.04 & 32839.35 & 21697 & 20.00 & 180 & 21897.00 & 10942.35 & 1.50 \\
\hline $\mathrm{T}_{4}$ & $\begin{array}{l}\text { Carbendazim } 50 \% \text { WP (ST) + Trichoderma viride } \\
\text { (ST) }\end{array}$ & $1 \mathrm{~g} / \mathrm{kg}$ seed $+12 \mathrm{~g} / \mathrm{kg}$ seed & $22.92(28.57)$ & 766.48 & 35449.7 & 21697 & 38.40 & 180 & 21915.40 & 13534.30 & 1.62 \\
\hline $\mathrm{T}_{5}$ & $\begin{array}{l}\text { (Carbendazim } 25 \% \mathrm{WP}+\text { mancozeb } 50 \% \mathrm{WP})+ \\
\text { Trichoderma viride }(\mathrm{ST})\end{array}$ & $3 \mathrm{~g} / \mathrm{kg}$ seed $+12 \mathrm{~g} / \mathrm{kg}$ seed & $21.25(27.39)$ & 780.20 & 36084.25 & 21697 & 48.00 & 180 & 21925.00 & 14159.25 & 1.65 \\
\hline $\mathrm{T}_{6}$ & Neem seed cake (SA) & $5 \mathrm{q} / \mathrm{ha}$ & $23.75(29.13)$ & 761.39 & 35214.29 & 21697 & 4175 & 260 & 26132.00 & 9082.29 & 1.35 \\
\hline $\mathrm{T}_{7}$ & Castor seed cake (SA) & $5 \mathrm{q} / \mathrm{ha}$ & $24.17(29.40)$ & 740.36 & 34241.65 & 21697 & 4350 & 260 & 26307.00 & 7934.65 & 1.30 \\
\hline $\mathrm{T}_{8}$ & Trichoderma viride (SA) & $20 \mathrm{~kg} / \mathrm{ha}$ & $21.25(27.39)$ & 793.04 & 36678.1 & 21697 & 687.50 & 180 & 22564.50 & 14113.60 & 1.63 \\
\hline $\mathrm{T}_{9}$ & Trichoderma viride $(\mathrm{SA})+$ neem seed cake $(\mathrm{SA})$ & $20 \mathrm{~kg} / \mathrm{ha}+5 \mathrm{q} / \mathrm{ha}$ & $17.50(24.66)$ & 912.11 & 42185.09 & 21697 & 4862.50 & 260 & 26819.50 & 15365.59 & 1.57 \\
\hline $\mathrm{T}_{10}$ & Trichoderma viride (SA) + castor seed cake (SA) & $20 \mathrm{~kg} / \mathrm{ha}+5 \mathrm{q} / \mathrm{ha}$ & $19.25(25.98)$ & 832.21 & 38489.71 & 21697 & 5037.50 & 260 & 26994.50 & 11495.21 & 1.43 \\
\hline $\mathrm{T}_{11}$ & $\begin{array}{l}\text { Trichoderma viride }(\mathrm{SA})+[\text { carbendazim } 25 \% \mathrm{WP} \\
+ \text { mancozeb } 50 \% \mathrm{WP} \text { ](ST) }\end{array}$ & $20 \mathrm{~kg} / \mathrm{ha}+3 \mathrm{~g} / \mathrm{kg}$ seed & $15.42(23.00)$ & 958.15 & 44314.44 & 21697 & 715.50 & 180 & 22592.50 & 21721.94 & 1.96 \\
\hline $\mathrm{T}_{12}$ & $\begin{array}{l}\text { T. v. (SA) + neem seed cake (SA) + [carbendazim } \\
25 \% \text { WP + mancozeb } 50 \% \text { WP] (ST) + T. v. (ST) }\end{array}$ & $\begin{array}{l}20 \mathrm{~kg} / \mathrm{ha}+5 \mathrm{q} / \mathrm{ha}+3 \mathrm{~g} / \\
\mathrm{kg} \mathrm{seed}+12 \mathrm{~g} / \mathrm{kg} \text { seed }\end{array}$ & $12.50(20.63)$ & 1000.65 & 46280.06 & 21697 & 4910.50 & 260 & 26867.50 & 19412.56 & 1.72 \\
\hline $\mathrm{T}_{13}$ & $\begin{array}{l}\text { T. v. }(\mathrm{SA})+\text { neem seed cake }(\mathrm{SA})+[\text { carbendazim } \\
+ \text { Mancozeb }](\mathrm{ST})+T . v .(\mathrm{ST})+[\text { carbendazim } 25 \\
\% \mathrm{WP}+\text { mancozeb } 50 \% \mathrm{WP}](\mathrm{SD})\end{array}$ & $\begin{array}{l}20 \mathrm{~kg} / \mathrm{ha}+5 \mathrm{q} / \mathrm{ha}+ \\
3 \mathrm{~g} / \mathrm{kg} \mathrm{seed}+12 \mathrm{~g} / \mathrm{kg} \text { seed } \\
+ \text { + } 0.25 \%\end{array}$ & $9.58(17.88)$ & 1109.10 & 51295.88 & 21697 & 5078.50 & 540 & 27315.50 & 23980.38 & 1.88 \\
\hline $\mathrm{T}_{14}$ & $\begin{array}{l}\text { T. v. (SA) + neem seed cake (SA)+ [carbendazim } \\
25 \% \mathrm{WP}+\text { mancozeb } 50 \% \mathrm{WP}](\mathrm{ST})+T . v .(\mathrm{ST}) \\
+ \text { propiconazole }(\mathrm{SD})\end{array}$ & $\begin{array}{l}20 \mathrm{~kg} / \mathrm{ha}+5 \mathrm{q} / \mathrm{ha}+ \\
3 \mathrm{~g} / \mathrm{kg} \mathrm{seed}+12 \mathrm{~g} / \mathrm{kg} \text { seed } \\
+@ 0.1 \%\end{array}$ & $7.13(15.18)$ & 1175.94 & 54387.23 & 21697 & 5217.70 & 540 & 27454.70 & 26932.53 & 1.98 \\
\hline $\mathrm{T}_{15}$ & $\begin{array}{l}\text { T. v. (SA) + neem seed cake (SA)+ [carbendazim } \\
25 \% \text { WP + mancozeb } 50 \% \text { WP }] \\
(\mathrm{ST})+\text { T.v. }(\mathrm{ST})+\text { thiophanate methyl }(\mathrm{SD})\end{array}$ & $\begin{array}{l}20 \mathrm{~kg} / \mathrm{ha}+5 \mathrm{q} / \mathrm{ha}+ \\
3 \mathrm{~g} / \mathrm{kg} \mathrm{seed}+12 \mathrm{~g} / \mathrm{kg} \text { seed } \\
+ \text { + } 0.1 \%\end{array}$ & $\begin{array}{c}5.00 \\
(12.00)\end{array}$ & 1347.48 & 62320.95 & 21697 & 5042.50 & 540 & 27279.50 & 35041.45 & 2.28 \\
\hline $\mathrm{T}_{16}$ & $\begin{array}{l}\text { T. } v .(\mathrm{SA})+\text { neem seed cake (SA)+ [carbendazim } \\
25 \% \text { WP + mancozeb } 50 \% \mathrm{WP}](\mathrm{ST})+T . v .(\mathrm{ST}) \\
+ \text { azoxystrobin } 23 \mathrm{EC}(\mathrm{SD})\end{array}$ & $\begin{array}{l}20 \mathrm{~kg} / \mathrm{ha}+5 \mathrm{q} / \mathrm{ha}+ \\
3 \mathrm{~g} / \mathrm{kg} \mathrm{seed}+12 \mathrm{~g} / \mathrm{kg} \text { seed } \\
+ \text { + } 0.1 \%\end{array}$ & $\begin{array}{c}3.33 \\
(8.33)\end{array}$ & 1424.28 & 65872.95 & 21697 & 5527.06 & 540 & 27764.06 & 38108.89 & 2.37 \\
\hline $\mathrm{T}_{17}$ & Control & Untreated & $\begin{array}{c}43.92 \\
(41.47)\end{array}$ & 582.33 & 26932.76 & 21697 & 00.00 & 00.00 & 21697.00 & 5235.76 & 1.24 \\
\hline \multicolumn{3}{|l|}{ S.E. \pm} & 2.00 & 66.12 & & & & & & & \\
\hline \multicolumn{3}{|c|}{ C.D. $(\mathbf{P}=0.05)$} & 5.81 & 191.35 & & & & & & & \\
\hline \multicolumn{3}{|l|}{ C.V. } & 14.00 & 12.65 & & & & & & & \\
\hline \multicolumn{12}{|c|}{$\begin{array}{l}*: \text { Mean of three replications, ^: Selling rates of pigeonpea seed yield @ 4625/q, Labour Charges: } 180 \text { Rs / labour, **: As per Annexure III (A), ***: As per costs } \\
\text { mentioned in the chapter III, ^^: Seed treatment and drenching charges @ Rs. 180/labour, ICBR: Incremental Cost Benefit Ratio, ST: Seed Treatment; SA: Soil } \\
\text { Application; SD: Soil Drenching. } \\
\text { Figures in parentheses are angular transformed values }\end{array}$} \\
\hline
\end{tabular}


Table.5 Economics of integrated management practices imposed for Fusarium wilt disease of pigeonpea during Kharif 2016-17

\begin{tabular}{|c|c|c|c|c|c|c|c|c|c|c|c|}
\hline \multirow{2}{*}{$\begin{array}{l}\text { Tr. } \\
\text { No }\end{array}$} & \multirow[b]{2}{*}{ Treatments } & \multirow[t]{2}{*}{ Rate of application } & \multirow[t]{2}{*}{ PDI (\%) } & \multirow{2}{*}{$\begin{array}{l}\text { Seed Yield* } \\
(\mathrm{kg} / \mathrm{ha})\end{array}$} & \multirow{2}{*}{$\begin{array}{c}\text { Gross } \\
\text { Returns }^{\wedge} \\
\text { (Rs / ha) }\end{array}$} & \multirow{2}{*}{$\begin{array}{l}\text { Cost of } \\
\text { Cultivatio } \\
\text { n (Rs / ha) }\end{array}$} & \multicolumn{2}{|c|}{ Cost Plant Protection (Rs / ha) } & \multirow{2}{*}{$\begin{array}{l}\text { Total Cost } \\
\text { (Rs / ha) }\end{array}$} & \multirow[t]{2}{*}{ Net Profit } & \multirow[t]{2}{*}{ ICBR } \\
\hline & & & & & & & $\underset{\substack{\text { Treat-mens } \\
* * *}}{ }$ & $\begin{array}{c}\text { Labour } \\
\text { Charges^^}\end{array}$ & & & \\
\hline 1 & 2 & 3 & 4 & 5 & 6 & 7 & 8 & 9 & 10 & 11 & 12 \\
\hline $\mathrm{T}_{1}$ & Carbendazim 50\%WP (ST) & $1 \mathrm{~g} / \mathrm{kg}$ seed & $\begin{array}{c}28.33 \\
(32.10)\end{array}$ & 685.91 & 34638.46 & 21697 & 18.40 & 180 & 21895.40 & 12743.06 & 1.58 \\
\hline $\mathrm{T}_{2}$ & Carbendazim $25 \% \mathrm{WP}+$ mancozeb $50 \% \mathrm{WP}(\mathrm{ST})$ & $3 \mathrm{~g} / \mathrm{kg}$ seed & $\begin{array}{c}27.62 \\
(31.66)\end{array}$ & 717.78 & 36247.89 & 21697 & 28.00 & 180 & 21905.00 & 14342.89 & 1.65 \\
\hline $\mathrm{T}_{3}$ & Trichoderma viride (ST) & $12 \mathrm{~g} / \mathrm{kg}$ seed & $\begin{array}{c}29.17 \\
(32.60)\end{array}$ & 662.23 & 33442.62 & 21697 & 20.00 & 180 & 21897.00 & 11545.62 & 1.53 \\
\hline $\mathrm{T}_{4}$ & $\begin{array}{l}\text { Carbendazim } 50 \% \text { WP (ST) + Trichoderma viride } \\
\text { (ST) }\end{array}$ & $\begin{array}{l}1 \mathrm{~g} / \mathrm{kg} \text { seed }+ \\
12 \mathrm{~g} / \mathrm{kg} \text { seed }\end{array}$ & $\begin{array}{c}26.25 \\
(30.79)\end{array}$ & 734.83 & 37108.92 & 21697 & 38.40 & 180 & 21915.40 & 15193.52 & 1.69 \\
\hline $\mathrm{T}_{5}$ & $\begin{array}{l}\text { (Carbendazim } 25 \% \text { WP + mancozeb } 50 \% \\
\text { WP)+T.viride (ST) }\end{array}$ & $\begin{array}{l}3 \mathrm{~g} / \mathrm{kg} \text { seed }+ \\
12 \mathrm{~g} / \mathrm{kg} \text { seed }\end{array}$ & $\begin{array}{c}25.00 \\
(29.93)\end{array}$ & 742.13 & 37477.57 & 21697 & 48.00 & 180 & 21925.00 & 15552.57 & 1.71 \\
\hline $\mathrm{T}_{6}$ & Neem seed cake (SA) & $5 \mathrm{q} / \mathrm{ha}$ & $\begin{array}{c}26.25 \\
(30.78)\end{array}$ & 721.10 & 36415.55 & 21697 & 4175 & 260 & 26132.00 & 10283.55 & 1.39 \\
\hline $\mathrm{T}_{7}$ & Castor seed cake (SA) & $5 \mathrm{q} / \mathrm{ha}$ & $\begin{array}{c}27.92 \\
(31.87)\end{array}$ & 692.77 & 34984.89 & 21697 & 4350 & 260 & 26307.00 & 8677.89 & 1.33 \\
\hline $\mathrm{T}_{8}$ & Trichoderma viride (SA) & $20 \mathrm{~kg} / \mathrm{ha}$ & $\begin{array}{c}24.26 \\
(29.45)\end{array}$ & 756.96 & 38226.48 & 21697 & 687.50 & 180 & 22564.50 & 15661.98 & 1.69 \\
\hline $\mathrm{T}_{9}$ & $\begin{array}{l}\text { Trichoderma viride }(\mathrm{SA})+ \\
\text { neem seed cake }(\mathrm{SA})\end{array}$ & $20 \mathrm{~kg} / \mathrm{ha}+5 \mathrm{q} / \mathrm{ha}$ & $\begin{array}{c}21.67 \\
(27.72)\end{array}$ & 853.68 & 43110.84 & 21697 & 4862.50 & 260 & 26819.50 & 16291.34 & 1.61 \\
\hline $\mathrm{T}_{10}$ & Trichoderma viride $(\mathrm{SA})+$ castor seed cake $(\mathrm{SA})$ & $20 \mathrm{~kg} / \mathrm{ha}+5 \mathrm{q} / \mathrm{ha}$ & $\begin{array}{c}22.31 \\
(28.11)\end{array}$ & 710.04 & 35857.02 & 21697 & 5037.50 & 260 & 26994.50 & 8862.52 & 1.33 \\
\hline $\mathrm{T}_{11}$ & $\begin{array}{l}\text { Trichoderma viride (SA) + [carbendazim } 25 \% \mathrm{WP} \\
+ \text { mancozeb } 50 \% \mathrm{WP} \text { ] (ST) }\end{array}$ & $\begin{array}{l}20 \mathrm{~kg} / \mathrm{ha}+3 \mathrm{~g} / \mathrm{kg} \\
\text { seed }\end{array}$ & $\begin{array}{c}19.25 \\
(25.96)\end{array}$ & 920.75 & 46497.88 & 21697 & 715.50 & 180 & 22592.50 & 23905.38 & 2.06 \\
\hline$T_{12}$ & $\begin{array}{l}\text { T. v. (SA) + neem seed cake (SA) +[carbendazim } \\
25 \% \text { WP+ mancozeb } 50 \% \text { WP] (ST) + T. v. (ST) }\end{array}$ & $\begin{array}{l}20 \mathrm{~kg} / \mathrm{ha}+5 \mathrm{q} / \mathrm{ha}+3 \\
\mathrm{~g} / \mathrm{kg} \text { seed }+ \\
12 \mathrm{~g} / \mathrm{kg} \text { seed }\end{array}$ & $\begin{array}{c}16.31 \\
(23.75)\end{array}$ & 969.22 & 48945.61 & 21697 & 4910.50 & 260 & 26867.50 & 22078.11 & 1.82 \\
\hline$T_{13}$ & $\begin{array}{l}T . v .(\mathrm{SA})+\text { neem seed cake }(\mathrm{SA})+\text { [carbendazim }+ \\
\text { mancozeb ] (ST) + T. v. (ST) + [carbendazim } 25 \% \\
\mathrm{WP}+\text { mancozeb } 50 \% \text { WP] }(\mathrm{SD})\end{array}$ & $\begin{array}{l}20 \mathrm{~kg} / \mathrm{ha}+5 \mathrm{q} / \mathrm{ha}+ \\
3 \mathrm{~g} / \mathrm{kg} \text { seed }+12 \mathrm{~g} / \mathrm{kg} \\
\text { seed +@ } 0.25 \%\end{array}$ & $\begin{array}{c}12.92 \\
(20.99)\end{array}$ & 1077.45 & 54411.23 & 21697 & 5078.50 & 540 & 27315.50 & 27095.73 & 1.99 \\
\hline $\mathrm{T}_{14}$ & $\begin{array}{l}\text { T. v. (SA) + neem seed cake (SA)+ [carbendazim } 25 \\
\% \text { WP + mancozeb } 50 \% \text { WP](ST) + T. v. (ST) + } \\
\text { propiconazole (SD) }\end{array}$ & $\begin{array}{l}20 \mathrm{~kg} / \mathrm{ha}+5 \mathrm{q} / \mathrm{ha}+3 \\
\mathrm{~g} / \mathrm{kg} \text { seed }+12 \mathrm{~g} / \mathrm{kg} \\
\text { seed }+@ 0.1 \%\end{array}$ & $\begin{array}{c}10.47 \\
(18.77)\end{array}$ & 1152.93 & 58222.97 & 21697 & 5217.70 & 540 & 27454.70 & 30768.27 & 2.12 \\
\hline $\mathrm{T}_{15}$ & $\begin{array}{l}\text { T. v. (SA) + neem seed cake (SA)+ [carbendazim } 25 \\
\% \text { WP + mancozeb } 50 \% \text { WP] }(\mathrm{ST})+\text { T. v. (ST) + } \\
\text { thiophanate methyl (SD) }\end{array}$ & $\begin{array}{l}20 \mathrm{~kg} / \mathrm{ha}+5 \mathrm{q} / \mathrm{ha}+3 \\
\mathrm{~g} / \mathrm{kg} \mathrm{seed}+12 \mathrm{~g} / \mathrm{kg} \\
\text { seed }+@ 0.1 \%\end{array}$ & $\begin{array}{c}9.17 \\
(17.56)\end{array}$ & 1336.85 & 67510.93 & 21697 & 5042.50 & 540 & 27279.50 & 40231.43 & 2.47 \\
\hline $\mathrm{T}_{16}$ & $\begin{array}{l}\text { T. v. (SA) + neem seed cake (SA)+ [carbendazim } 25 \\
\% \text { WP + mancozeb } 50 \% \text { WP](ST) + T. v. (ST) + } \\
\text { azoxystrobin } 23 \text { EC (SD) }\end{array}$ & $\begin{array}{l}20 \mathrm{~kg} / \mathrm{ha}+5 \mathrm{q} / \mathrm{ha}+3 \\
\mathrm{~g} / \mathrm{kg} \mathrm{seed}+12 \mathrm{~g} / \mathrm{kg} \\
\text { seed }+@ 0.1 \%\end{array}$ & $\begin{array}{c}7.12 \\
(15.37)\end{array}$ & 1368.73 & 69120.87 & 21697 & 5527.06 & 540 & 27764.06 & 41356.81 & 2.49 \\
\hline $\mathrm{T}_{17}$ & Control & Untreated & $\begin{array}{c}48.75 \\
(44.26)\end{array}$ & 541.82 & 27361.91 & 21697 & 00.00 & 00.00 & 21697.00 & 5664.91 & 1.26 \\
\hline \multicolumn{3}{|c|}{ S.E. \pm} & 1.47 & 50.61 & & & & & & & \\
\hline \multicolumn{3}{|c|}{ C.D. $(\mathbf{P}=0.05)$} & 4.26 & 146.44 & & & & & & & \\
\hline \multicolumn{3}{|l|}{ C.V. } & 9.19 & 10.17 & & & & & & & \\
\hline
\end{tabular}

*: Mean of three replications, ^: Selling rates of pigeonpea seed yield @ 5050/q, Labour Charges: 180 Rs/labour, **: As per Annexure III (A), ***: As per costs mentioned in the chapter III, $\wedge$ : Seed treatment and drenching charges @ Rs. 180/labour,

ICBR: Incremental Cost Benefit Ratio; ST: Seed Treatment; SA: Soil Application; SD: Soil Drenching.

Figures in parentheses are angular transformed values 
Table.6 Pooled mean of incremental Cost: Benefit ratio (Kharif 2015-16 and Kharif 2016-17)

\begin{tabular}{|c|c|c|c|c|c|}
\hline \multirow{2}{*}{$\begin{array}{l}\text { Tr. } \\
\text { No. }\end{array}$} & \multirow[t]{2}{*}{ Treatments } & \multirow[t]{2}{*}{ Rate of application } & \multicolumn{2}{|c|}{ ICBR } & \multirow{2}{*}{$\begin{array}{l}\text { Pooled } \\
\text { Mean }\end{array}$} \\
\hline & & & 2015-16 & 2016-17 & \\
\hline $\mathrm{T}_{1}$ & Carbendazim $50 \%$ WP (ST) & $1 \mathrm{~g} / \mathrm{kg}$ seed & 1.55 & 1.58 & 1.57 \\
\hline $\mathrm{T}_{2}$ & $\begin{array}{l}\text { Carbendazim } 25 \% \text { WP }+ \\
\text { mancozeb } 50 \% \text { WP }(\mathrm{ST})\end{array}$ & $3 \mathrm{~g} / \mathrm{kg}$ seed & 1.6 & 1.65 & 1.63 \\
\hline $\mathrm{T}_{3}$ & Trichoderma viride $(\mathrm{ST})$ & $12 \mathrm{~g} / \mathrm{kg}$ seed & 1.5 & 1.53 & 1.52 \\
\hline $\mathrm{T}_{4}$ & $\begin{array}{l}\text { Carbendazim } 50 \% \text { WP (ST) } \\
+T . \text { viride (ST) }\end{array}$ & $\begin{array}{l}1 \mathrm{~g} / \mathrm{kg} \text { seed }+ \\
12 \mathrm{~g} / \mathrm{kg} \text { seed }\end{array}$ & 1.62 & 1.69 & 1.66 \\
\hline $\mathrm{T}_{5}$ & 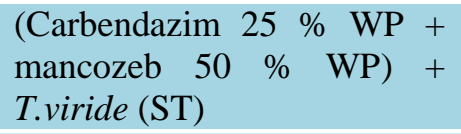 & $\begin{array}{l}3 \mathrm{~g} / \mathrm{kg} \text { seed }+ \\
12 \mathrm{~g} / \mathrm{kg} \text { seed }\end{array}$ & 1.65 & 1.71 & 1.68 \\
\hline $\mathrm{T}_{6}$ & Neem seed cake (SA) & $5 \mathrm{q} / \mathrm{ha}$ & 1.35 & 1.39 & 1.37 \\
\hline $\mathrm{T}_{7}$ & castor seed cake (SA) & $5 \mathrm{q} / \mathrm{ha}$ & 1.3 & 1.33 & 1.32 \\
\hline $\mathrm{T}_{8}$ & T. viride (SA) & $20 \mathrm{~kg} / \mathrm{ha}$ & 1.63 & 1.69 & 1.66 \\
\hline $\mathrm{T}_{9}$ & $\begin{array}{l}\text { T. viride }(\mathrm{SA})+\text { neem seed } \\
\text { cake (SA) }\end{array}$ & $20 \mathrm{~kg} / \mathrm{ha}+5 \mathrm{q} / \mathrm{ha}$ & 1.57 & 1.61 & 1.59 \\
\hline $\mathrm{T}_{10}$ & $\begin{array}{l}\text { T. viride }(\mathrm{SA})+\text { castor seed } \\
\text { cake }(\mathrm{SA})\end{array}$ & $20 \mathrm{~kg} / \mathrm{ha}+5 \mathrm{q} / \mathrm{ha}$ & 1.43 & 1.33 & 1.38 \\
\hline $\mathrm{T}_{11}$ & $\begin{array}{l}T . \quad \text { viride }(\mathrm{SA}) \\
{[\text { Carbendazim } 25 \% \mathrm{WP}+} \\
\text { mancozeb } 50 \% \mathrm{WP}](\mathrm{ST})\end{array}$ & $\begin{array}{l}20 \mathrm{~kg} / \mathrm{ha}+3 \mathrm{~g} / \mathrm{kg} \\
\text { seed }\end{array}$ & 1.96 & 2.06 & 2.01 \\
\hline $\mathrm{T}_{12}$ & $\begin{array}{l}\text { T.v. }(\mathrm{SA})+\text { neem seed cake } \\
(\mathrm{SA})+[\text { Carbendazim } 25 \% \\
\mathrm{WP}+\text { mancozeb } 50 \% \mathrm{WP}] \\
(\mathrm{ST})+\text { T.v. }(\mathrm{ST})\end{array}$ & $\begin{array}{l}20 \mathrm{~kg} / \mathrm{ha}+5 \mathrm{q} / \mathrm{ha}+ \\
3 \mathrm{~g} / \mathrm{kg} \text { seed }+12 \mathrm{~g} / \\
\mathrm{kg} \text { seed }\end{array}$ & 1.72 & 1.82 & 1.77 \\
\hline $\mathrm{T}_{13}$ & $\begin{array}{l}\text { T.v. }(\mathrm{SA})+ \\
\text { neem seed cake (SA) + } \\
{[\text { Carbendazim }+ \text { mancozeb }]} \\
(\mathrm{ST})+\text { T.v. }(\mathrm{ST})+ \\
{[\text { carbendazim } 25 \% \text { WP }+} \\
\text { mancozeb } 50 \% \mathrm{WP}](\mathrm{SD})\end{array}$ & $\begin{array}{l}20 \mathrm{~kg} / \mathrm{ha}+ \\
5 \mathrm{q} / \mathrm{ha}+ \\
3 \mathrm{~g} / \mathrm{kg} \text { seed }+ \\
12 \mathrm{~g} / \mathrm{kg} \text { seed }+@ \\
0.25 \%\end{array}$ & 1.88 & 1.99 & 1.94 \\
\hline $\mathrm{T}_{14}$ & $\begin{array}{l}\text { T.v. }(\mathrm{SA})+\text { neem seed cake } \\
(\mathrm{SA})+\text { [carbendazim } 25 \% \\
\mathrm{WP}+\text { mancozeb } 50 \% \mathrm{WP}] \\
(\mathrm{ST})+\text { T.v. } \quad(\mathrm{ST})+ \\
\text { propiconazole }(\mathrm{SD})\end{array}$ & $\begin{array}{l}20 \mathrm{~kg} / \mathrm{ha}+ \\
5 \mathrm{q} / \mathrm{ha}+ \\
3 \mathrm{~g} / \mathrm{kg} \text { seed }+ \\
12 \mathrm{~g} / \mathrm{kg} \text { seed } \\
+@ 0.1 \%\end{array}$ & 1.98 & 2.12 & 2.05 \\
\hline $\mathrm{T}_{15}$ & $\begin{array}{l}\text { T.v. }(\mathrm{SA})+\text { neem seed cake } \\
\text { (SA)+ [carbendazim } 25 \% \\
\mathrm{WP}+\text { mancozeb } 50 \% \mathrm{WP}] \\
(\mathrm{ST})+\text { T.v. (ST) } \\
\text { thiophanate methyl (SD) }\end{array}$ & $\begin{array}{l}20 \mathrm{~kg} / \mathrm{ha}+ \\
5 \mathrm{q} / \mathrm{ha}+ \\
3 \mathrm{~g} / \mathrm{kg} \mathrm{seed}+ \\
12 \mathrm{~g} / \mathrm{kg} \text { seed }+ \\
\text { @ } 0.1 \%\end{array}$ & 2.28 & 2.47 & 2.38 \\
\hline $\mathrm{T}_{16}$ & $\begin{array}{l}\text { T.v. }(\mathrm{SA})+\text { neem seed cake } \\
(\mathrm{SA})+\text { [carbendazim } 25 \% \\
\mathrm{WP}+\text { mancozeb } 50 \% \mathrm{WP}] \\
(\mathrm{ST})+\text { T.v. }(\mathrm{ST})+ \\
\text { azoxystrobin } 23 \mathrm{EC}(\mathrm{SD})\end{array}$ & $\begin{array}{l}20 \mathrm{~kg} / \mathrm{ha}+ \\
5 \mathrm{q} / \mathrm{ha}+ \\
3 \mathrm{~g} / \mathrm{kg} \mathrm{seed}+ \\
12 \mathrm{~g} / \mathrm{kg} \text { seed } \\
+@ 0.1 \%\end{array}$ & 2.37 & 2.49 & 2.43 \\
\hline $\mathrm{T}_{17}$ & Control & Untreated & 1.24 & 1.26 & 1.25 \\
\hline
\end{tabular}

ST: Seed Treatment; SA: Soil Application; SD: Soil Drenching 
$\mathrm{T}_{16}$ treatment showed maximum per cent reduction of wilt (92.42 and $59.17 \%$ ) over control in normal as well as sick soil, respectively followed by treatment $\mathrm{T}_{15}$ (88.62 and $56.07 \%$ ). It was minimum in $\mathrm{T}_{3}$ : Seed treatment of Trichoderma viride $12 \mathrm{~g} / \mathrm{kg}$ of seed (37.39 and $12.92 \%$ ) during Kharif 2015-16

Efficacy of various treatments integration against wilt ( $F$. udum) incidence and seed yield during Kharif 2016-17

Under normal soil and sick soil conditions, $\mathrm{T}_{16}$ [soil application of $(T . v$. + neem seed cake) + seed treatment of (carbendazim $25 \% \mathrm{WP}+$ mancozeb $50 \% \mathrm{WP}+T$. v. $)+$ soil drenching of azoxystrobin $23 \mathrm{EC}]$ showed minimum wilt incidence i.e. 7.12 and $45.42 \%$ with maximum yield i.e. 1368.73 and $458.38 \mathrm{~kg} / \mathrm{ha}$, respectively compared to other treatments.

Second best treatment was $\mathrm{T}_{15}$ [soil application of $(T . v .+$ neem seed cake $)+$ seed treatment of (carbendazim $25 \% \mathrm{WP}+$ mancozeb $50 \% \mathrm{WP}$ $+T$. v.) + soil drenching of thiophanate methyl] which showed $9.17 \%$ wilt incidence with $1336.85 \mathrm{~kg} / \mathrm{ha}$ yield in normal soil and in sick soil it was $47.08 \%$ with $443.77 \mathrm{~kg} / \mathrm{ha}$ yield. Maximum wilt incidences (48.75 and $100 \%$ ) were recorded in untreated control $\mathrm{T}_{17}$ with $541.82 \mathrm{~kg} / \mathrm{ha}$ yield and without any yield in normal and sick soil, respectively (Table 2).

$\mathrm{T}_{16}$ treatment showed maximum per cent reduction of ( 85.39 and $54.58 \%$ ) over untreated control in normal and sick soil, respectively followed by treatment $\mathrm{T}_{15}(81.19$ and $52.92 \%)$. It was minimum in $\mathrm{T}_{3}$ : Seed treatment of Trichoderma viride $12 \mathrm{~g} / \mathrm{kg}$ of seed (40.16 and $08.75 \%$ ) during Kharif 2016-17 (Table 2).

These results are in conformity with the findings of those reported earlier by several workers (Gade et al., 2007; Dabbas et al., 2008; Mahesh et al., 2010; Karimi et al., 2012; Prasad et al., 2012; Chennakesavulu et al., 2013; Pawar et al., 2013; Kumar and Upadhayay 2015).

\section{Pooled means of wilt incidence and seed yield} (IDM)

The pooled results indicated that pooled mean of wilt incidence and seed yield $(\mathrm{kg} / \mathrm{ha})$ were significantly influenced with various treatments imposed to manage wilt incidence and 562.08 to $1396.50 \mathrm{~kg} / \mathrm{ha}$ respectively, in normal soil, where as in sick soil wilt incidence was ranged from $43.13\left(\mathrm{~T}_{16}\right)$ to $100 \%$ and yield ranged from 0 to $485.72 \mathrm{~kg} / \mathrm{ha}$. Result of pooled analysis showed non-significant interaction of two years and seventeen treatments for both soil conditions but during Kharif 2015-16 and 201617 all the treatments were reduced the wilt incidence and increase the yield compare to untreated control under normal and sick soil conditions (Table 3).

\section{Pooled mean of incremental Cost: Benefit ratio (under normal soil)}

Results obtained during, Kharif 2015-16 and 2016-17 on economics / incremental cost: benefit (ICBR) in respect of the treatments integrated to manage pigeonpea wilt disease revealed that all the treatments significantly increased the seed yield, with maximum net profit and increased ICBR as compared to untreated control during both the years.

Among various treatments, [soil application of $(T$. $v$. + neem seed cake $)+$ seed treatment of (carbendazim $25 \% \mathrm{WP}+$ mancozeb $50 \% \mathrm{WP}$ $+T$. v. $)+$ soil drenching of azoxystrobin $23 \mathrm{EC}]$ was found most effective, with the highest gross return (Rs. 65872.95/- and 69120.87/-), highest net profit (Rs.38108.89/- and 41356.81/-) and highest ICBR (2.37 and 2.49) during Kharif 2015-16 and 2016-17, respectively (Table 5).

The second best treatment found was [soil application of $(T . v .+$ neem seed cake $)+$ seed treatment of (carbendazim $25 \% \mathrm{WP}+$ mancozeb $50 \% \mathrm{WP}+T . v$.) + soil drenching of thiophanate methyl] with second highest gross income (Rs.62320.95/- and 67510.93/-), net profit (Rs. 35041.45/- and 40231.43/-) and ICBR (2.28 and 2.47) during, Kharif 2015-16 
and 2016-17, respectively. Results indicated that on the basis of two years (2015-16 and 2016-17) pooled mean data, the most economical treatment with highest mean ICBR (2.43) was [soil application of $(T . v .+$ neem seed cake) + seed treatment of (carbendazim 25 $\% \mathrm{WP}+$ mancozeb $50 \% \mathrm{WP}+$ T. v. $)+$ Soil drenching of azoxystrobin 23 EC] (Table 6) followed by the treatment $\mathrm{T}_{15}$ [soil application of (T. v. + neem seed cake) + seed treatment of (carbendazim $25 \% \mathrm{WP}+$ mancozeb $50 \% \mathrm{WP}$ $+T$. v.) + soil drenching of thiophanate methyl] with the ICBR (2.38). The lowest ICBR (1.25) was recorded in treatment $\mathrm{T}_{17}$ (untreated control).

\section{References}

Butler, E. J. (1910). The wilt disease of pigeonpea and the parasitism of Neocosmospora vasinfecta. Department of Agriculture India. (Botany Section) 2: $1-62$.

Chennakesavulu, M., Reddi Kumar, M. and Eswara Reddy, N. P. (2013). Mass multiplicaction and self-life studies of Pseudomonas fluorescens against pigeonpea wilt. Indian $\mathrm{J}$. Pl. Protec. 41 (1): 45-49.

Dabbas, M. R., Srivastava, J. P. and Rai, M. (2008). Integrated Disease Management of Table pea. Ann. Pl. Protec. Sci. 16 (1): 156-158.

Gade, R. M., Zote, K. K. and Mayee, C. D. (2007). Integrated management of pigeonpea wilt using fungicide and bioagent. Indian phytopath, 60 (1): 24-30

Kannaiyan, J. and Nene Y. L. (1981). Influence of wilt at different growth stages on yield loss in pigeonpea. Trop. J. Pest Management, 27: 141.
Kannaiyan, J., Nene, Y. L., Reddy, M. V., Rayan, J. G. and Raju, T. N. (1984). Prevalence of pigeonpea diseases and associated crop losses in Asia and Amirica. Trop. J. Pest Management, 30: 62-71.

Karimi, R., James, O. O. and Silim, S. N. (2012). Importance and management of Fusarium wilt (Fusarium udum Butler) of pigeonpea. Inter. J. Agronomy Agril. Res., 2 (1): $1-14$.

Kumar, S. and Upadhyay, J. P. (2015). Development and validation of Integrated Disease Management module for Management of pigeonpea wilt caused by Fusarium udum. Indian J. Pl. Protec. 43 (4): 493-497.

Mahesh, M., Saifulla, M., Prasad, P. S. and Sreenivasa, S. (2010). Studies on cultural variability of Fusarium udum isolates in India. Inter. J. Sci. Nature 1 (2): 219225.

Nene, Y. L., Sheila, V. K. and Sharma, S. B. (1996). A world list of chickpea and pigeonpea pathogens (fifth edition) Patancheru, A.P., India. Int. crops Res. Inst. in Semi-Arid Tropics. pp. 27.

Pawar S. V., Deshpande, G. D., Dhutraj, D. N. and Dey, U. (2013). Survey of pigeonpea wilt disease in Marathwada region of Maharashtra state. A Quarterly J. life Sci., 10 (1): 175-176.

Prasad, P. S., Saifulla, M., Mallikarjuna, N., Thimmegowda, P. R. and Lakshmipathy, R. N. (2012). Integrated disease management of Pigeonpea wilt Fusarium udum (Butler). Madras Agric. J., 99 (10): 811-814.

\section{How to cite this article:}

Ghante, P.H., A.P. Suryawanshi, K.M. Kanase, S.D. Somwanshi and Thaware, D.S. 2018. Integrated Disease Management against Wilt Disease of Pigeonpea Caused by Fusarium oxysporum f. sp. udum. Int.J.Curr.Microbiol.App.Sci. 7(10): 2123-2132. doi: https://doi.org/10.20546/ijcmas.2018.710.244 